\title{
Metropolitan Wi-Fi Research Network at the Los Angeles State Historic Park
}

\author{
Vidyut Samanta \\ vids@remap.ucla.edu \\ Chase Laurelle Alexandria Knowles \\ chase@remap.ucla.edu \\ Jeff Burke \\ jburke@remap.ucla.edu \\ Fabian Wagmister \\ fabian@remap.ucla.edu \\ Deborah Estrin \\ destrin@,cs.ucla.edu \\ Center for Research in Engineering, Media and Performance, UCLA, Los Angeles, USA
}

\section{Introduction}

The Center for Research in Engineering, Media and Performance (REMAP) at the University of California at Los Angeles (UCLA), has collaborated with the NSF Center for Embedded Networked Sensing (CENS) to deploy a metropolitan scale Wi-Fi mesh network in the Los Angeles State Historic Park near Downtown Los Angeles. We refer to this network as Met-Wi. This will be an extension of the University's network into the communities around the park and will support research in community based urban participatory sensing [1], [2], [3], [4] through CENS and Cultural Civic Computing through REMAP.

Participatory Sensing is a new area of research at UCLA and focuses on how people can use their everyday mobile phones as sensors for data gathering on personal, community, and urban scales. Our Met-Wi deployment provides high-speed connectivity in certain areas for test devices, and a platform on which we can test network services in support of user participation in sensing.

REMAP and CENS recognize the significant role that community-based data collection can play in public health, urban planning and cultural applications, as well as the amazing potential for participation given the installed base of mobile phones. Cultural Civic Computing [7] is a service-oriented urban computing model in which communities power the processes of imagining, specifying, and designing technology infrastructure for public places. In this model, communities are involved in designing their infrastructure and the services it provides. Our Met-Wi deployment allows us to test out this approach. 


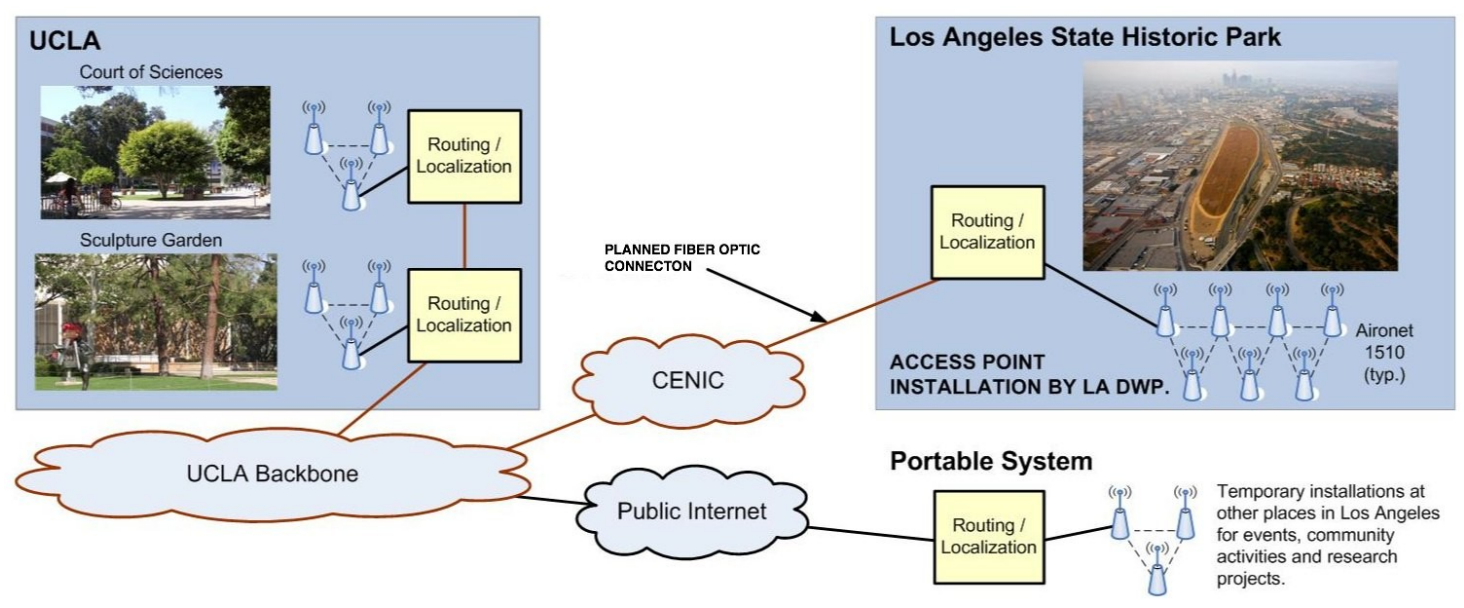

Figure 1: Met-Wi topology.

We are developing a web-based framework that will enable anyone with a Wi-Fi enabled device such as a mobile phone to

1. describe a "campaign" - i.e. a geographically and temporally constrained series of systematic operations to gather a particular type of data using a network of mobile devices (e.g., geotagged audio level measurements, a common quality of life metric in urban planning), and

2. recruit participants interested in the campaign,

3. who then engage in network-supported distributed data collection,

4. and are able to analyze and publish the results online,

5. and iterate to learn more.

We are interested in the network architecture needed in such a scenario to increase individuals' credibility as data providers to the community and encourage data sharing while protecting privacy. 


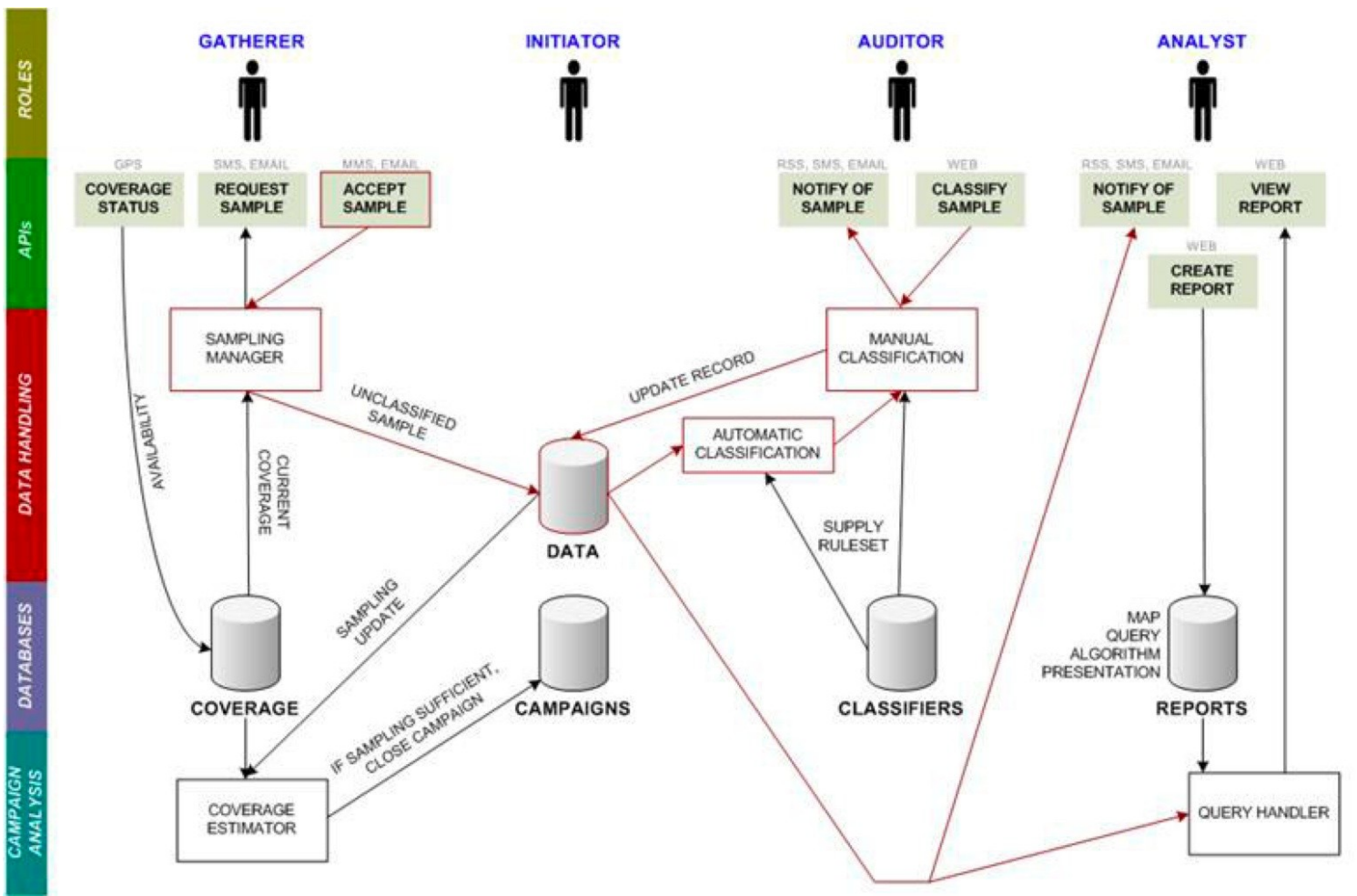

Figure 2: Campaign Framework

The Wi-Fi network will also have a location awareness component. Using RFFingerprinting technology that is part of the Cisco equipment, we will be able to geographically locate clients connected to the Wi-Fi network, and geotag data provided by clients with this location. Place-specific services are important to both of our application domains.

\section{Research}

Experiments will be performed in network support of community-based data gathering and documentary, interface design for diverse cultures, community mapping of geospatial data, and the creation of mobile and location-based multimedia experiences in the Park that interweave Los Angeles history with the local communities' self-expressed cultures and identities. These will seed larger-scale research projects with the community that could have significant impact in many fields. The next two sections describe some experiments and processes that REMAP and CENS have already begun.

\section{Urban \& Participatory Sensing}

The CENS urban sensing group is working on technologically-focused experiments using the campaign framework described above. For example, they are developing a sound level mapping campaign that aims to decipher the amount of noise pollution in and around the Park area. This campaign uses Wi-Fi enabled mobile phones to gather data samples of an arbitrary length of time that are then uploaded to a central server. The samples are processed to produce a sound pressure level estimate in decibels that is associated with the network-attested time and location where uploaded. This data will then be used to create a sound map of Los Angeles that useful to urban planners and developers. One application would be to use this process as a metric for the quality of life in Los Angeles. More 
generally, CENS is using the WiFi deployment to support a variety of projects enabling people to understand and explore their activities relative to communities and the urban environment, including projects such as a "Personal Environmental Impact Report" based on location trace information. In a related project, students are exploring whether geotagged photographs can be analyzed to estimate daily air pollution by using measures of visibility that can be extracted from the image.

\section{Cultural Civic Computing}

REMAP is focused on Cultural Civic Computing - on how emerging technologies can be re-envisioned by communities and researchers to enable public exploration of creativity, cultural identity, and the diverse histories of our cities and neighborhoods. Through collaborative experimentation with local residents and direct engagement with technology research programs, it aims to increase participation in community investigation of the city, collective remembering, self-representation, and imagining and defining the future of the urban environment. For instance, community members could use a variation of the sound mapping application above to create experiential soundscapes of their neighborhoods in addition to the level map. These explorations into Cultural Civic Computing are developing simultaneously with advances in personal and community GIS elsewhere, and we see great potential for interweaving them in the future.

REMAP has also developed prototype location-aware applications using the MetWi network. For example, REMAP has developed an extensive database of historic media about Los Angeles and the State Historic Park. This "geotagged" content is delivered to visitors while walking in the Park with Wi-Fi and location-enabled cell phones, allowing a fascinating exploration of the area's socio-historical significance.

\section{Sampling of Stakeholders}

CENS and REMAP are collaborating with the California Department of Parks and Recreation, local community organizations, and industry to explore participatory sensing and Cultural Civic Computing in and around the Los Angeles State Historic Park, using the network deployment described above.

\section{California Department of Parks and Recreation}

As this technology research was developing, REMAP established a formal partnership with California State Parks for the purpose of applying new technology towards communal investigation of local history, as well as issues of contemporary life, in the new Los Angeles State Historic Park; the Park is a 32-acre site outside of Downtown Los Angeles. Both California State Parks and UCLA are eager to work together to explore how emerging technologies can be used to provide new interpretive experiences in their parks especially in this unique urban setting.

\section{Local Communities}

The communities surrounding the Los Angeles State Historic Park collaborated to save this location from industrial development. During the lobbying process, a uniquely diverse neighborhood alliance - encompassing Chinatown, Solano Canyon, and Lincoln Heights - took root. These communities' role in helping create the Park has only added to the site's relevance as a historical record of Angeleno growth since the 1800s. In light of this sector's extensive involvement with the site, both State Parks and UCLA are committed to a research process at the Park, which deeply involves local communities. 
REMAP and CENS are collaborating with Cisco Systems [5] for the network infrastructure and, thanks to Nokia's provision of GSM and 802.11 mobile phones, are pursuing documentary and data collection for community use [6].

REMAP has also established unique, multidisciplinary partnerships with Walt Disney Imagineering Research \& Development and Schematic, a leading interactive design firm. With Disney Imagineering $R \& D$, we are collaborating on a temporary large-scale experiential installation located within the Park, which explores public experience of community history and identity, and is supported by connectivity from the Met-Wi deployment. Similarly, REMAP and CENS are cooperating with Schematic to engineer mobile interface design, which adequately supports community-based data gathering and documentation.

\section{Conclusion}

A metropolitan scale Wi-Fi mesh network is being deployed near Downtown Los Angeles in a collaborative effort of The Center for Research in Engineering, Media and Performance (REMAP) at UCLA, and the NSF Center for Embedded Networked Sensing (CENS). This work will help realize the potential a large-scale Wi-Fi deployment could offer a multicultural society for communal documentation of its surrounding environments. This will aid in the creation of healthy and livable cities, as well as enable public exploration of creativity and cultural identity, in conjunction with a greater appreciation of the diverse histories of our cities and neighborhoods.

\section{Acknowledgements}

In addition to support from Nokia, Cisco Systems, Walt Disney Imagineering Research and Development, Schematic, and collaboration with the California Dept. of Parks and Recreation, this material is based upon work supported by the NSF under award CNS-0627084. Any opinions, findings and conclusions or recommendations expressed in this material are those of the author(s) and do not necessarily reflect the views of the National Science Foundation.

\section{References}

Srivastava, M. and Hansen, M. and Burke, J. and Parker, A. and Reddy, S. and Saurabh, G. and Allman, M. and Paxson, V. and Estrin, D., Wireless Urban Sensing SystemsTechnical Report 65, Center for Embedded Networked Sensing Systems, UCLA, Apr. 2006.

Burke, J. and Estrin, D. and Hansen, M. and Parker, A. and Ramanathan, N. and Reddy, S. and Srivastava, MB, Participatory Sensing, World Wide Sensor Web Workshop, Sensys, 2006.

Parker, A. and Reddy, S. and Schmid, T. and Chang, K. and Saurabh, G. and Srivastava, M. and Hansen, M. and Burke, J. and Estrin, D. and Allman, M., Network System Challenges in Selective Sharing and Verification for Personal, Social, and Urban-Scale Sensing Applications, HotNets, 2006.

Reddy, S. and Parker, A. and Burke, J. and Estrin, D. and Hansen, H., Image Browsing, Processing, and Clustering for Participatory Sensing: Lessons From a DietSense Prototype, EmNets, 2007 
Cisco Systems Inc. Retrieved 30 July $2007<\underline{\text { http://www.cisco.com } />}$

Nokia SensorPlanet. Retrived 30 July $2007<\underline{\text { http://www.sensorplanet.org/> }}$

REMAPPING LA: Cultural Civic Computing in Los Angeles. Retrived 30 July 2007 $<\underline{\text { http://remap.ucla.edu/losangeles/ }>}$ 